\title{
Antimicrobial Drug Resistance Pattern of Escherichia coli Isolated from Chickens Farms with Colibacillosis Infection
}

\author{
Mohammad Jahantigh ${ }^{*}$, Reza Esmailzade Dizaji² \\ ${ }^{1}$ Department of Poultry Diseases, School of Veterinary Medicine, University of Zabol, Zabol, Iran \\ ${ }^{2}$ School of Veterinary Medicine, University of Zabol, Zabol, Iran \\ Email: "mjahantig@yahoo.com
}

Received 28 August 2015; accepted 22 November 2015; published 25 November 2015

Copyright (C) 2015 by authors and Scientific Research Publishing Inc.

This work is licensed under the Creative Commons Attribution International License (CC BY). http://creativecommons.org/licenses/by/4.0/

(c) (i) Open Access

\section{Abstract}

Colibacillosis refers to any localized or systemic infection caused entirely or partly by avian pathogenic Escherichia coli (APEC). Colibacillosis in mammals is most often a primary enteric or urinary tract disease, whereas colibacillosis in poultry is typically a localized or systemic disease occurring secondarily when host defenses have been impaired or overwhelmed by virulent $E$. coli strains. The purpose of this study was to investigate the antimicrobial drug resistance pattern of Escherichia coli isolated from broiler chickens farms with colibacillosis infection. Dead birds from commercial broiler chicken farms showing signs of colibacillosis were necropsied and swab samples were collected from internal organs and blood aseptically for the isolation of Escherichia coli. Pure colonies of the bacteria were isolated on solid media and the isolates were identified as $E$. coli based on morphological and biochemical characteristics. For determination of susceptibility to antibacterial agents, the disc diffusion method on Muller-Hinton agar was used. The following antimicrobial agents were tested: gentamycin, oxytetracyline, colistin, ciprofloxacin, doxycycline, nalidixic acid, co-trimoxazole (trimethoprim-sulfamethoxazole), norefloxacin, lincospectin and cefuroxime. The drug resistance patterns of the organisms were determined as a percentage and reported at three levels: susceptible, intermediate and resistant. All the isolates of Escherichia coli showed resistance to several antibiotics and a pattern of multiple drug resistance was observed. The highest rate of resistance was observed against nalidixic acid (100\%) and the least rate of resistance was observed against gentamycin $(17 \%)$. According to the results of this research care must be taken to avoid secondary infection (colibacillosis) in chicken farms and also avoid in careless antimicrobial consumption in food animals including chickens.

\footnotetext{
${ }^{*}$ Corresponding author.
}

How to cite this paper: Jahantigh, M. and Dizaji, R.E. (2015) Antimicrobial Drug Resistance Pattern of Escherichia coli Isolated from Chickens Farms with Colibacillosis Infection. Open Journal of Medical Microbiology, 5, 159-162. 


\section{Keywords}

\section{Chickens, Colibacillosis, Drug Resistance, Escherichia coli}

\section{Introduction}

Infection caused by Escherichia coli is known as colibacillosis. Airsacculitis, pericarditis, peritonitis, salpingitis, synovitis, osteomyelitis, cellulitis, coligranuloma, colisepticaemia and yolk sac infection are syndrome of $E$. coli infection [1]. Escherichia coli is the type genous of the family Enterobacteriaceae and grows aerobically or anaerobically on ordinary nutrient media at temperature $18^{\circ} \mathrm{C}-44^{\circ} \mathrm{C}$. It ferments carbohydrates, often producing gas [2]. Organisms are easily isolated and identified for confirmation of the disease. All broad-spectrum antibiotics respond to the infection [3]. Isolation of E. coli is significant if made from the internal organs or blood from fresh carcasses [4]. Escherichia coli, Enterobacter, and Klebsiella ferment lactose and can be distinguished from most of the other enteric organisms [4] [5]. Most avian pathogenic Escherichia coli (APEC) isolated from poultry are specific clonal types that are pathogenic only for birds and represents a low risk of disease for people or other animals. However, chickens are readily infected experimentally with E. coli O157:H7, an important enterohemorrhgic pathogen of humans that produces Shiga toxin, and can shed the organism for months. Natural infection with O157:H7 has been identified in both chickens and turkeys in different geographic areas. Contamination of poultry meat with this organism can occur as evidenced by a food-borne outbreak of diarrheal disease that was associated with contaminated turkey meat. Poultry, especially pigeons in certain geographic areas, are a natural reservoir for Shiga toxin producing E. coli (STEC) which is a potential health hazard to people [2]. Various antimicrobials in intensively managed food animals including chickens are often administered through feed or drinking water either for therapy, prophylaxis, or growth promotion [6]. High prevalence of resistance to antimicrobial agents amongst isolates of $E$. coli isolated from chickens has been reported [7]-[9].

The objectives of the present study were to investigate the antimicrobial resistance pattern of Escherichia coli isolated from broiler chickens farms with colibacillosis infection.

\section{Materials and Methods}

Dead birds from commercial broiler chickens farms in Zabol, southeast of Iran, showing signs of Escherichia coli infection (colibacillosis) were necropsied and swab samples were collected from internal organs and blood aseptically for the isolation of Escherichia coli. Swab samples were collected in $5 \mathrm{ml}$ Tryptone Soya Broth (TSB) and were transferred to the laboratory of microbiology and were incubated at $37^{\circ} \mathrm{C}$ for $18-24$ hours and were investigated for the presence of Escherichia coli. A total of $100 \mathrm{E}$. coli were isolated from chickens with fibrinous prehepatitis, precarditis, airsaculitis and yolk sac infection. Pure cultures were prepared from E. coli like colonies on Mac Conkey agar and Eosin Methylene Blue (EMB) agar (Merck, Germany). All isolates were identified as E. coli based on morphological and biochemical characteristics.

\section{Antimicrobial Susceptibility Testing}

For determination of susceptibility to antibacterial agents, the disc diffusion method on Muller-Hinton agar was used. Some colonies of isolated bacteria from an agar plate were transferred to a glass tube containing $5 \mathrm{ml}$ saline and the suspension was vortexed and visually matched with 0.5 MacFarland standard for turbidity. Sterile cotton tipped swab was dipped into the prepared bacterial suspension and then rolled on the upper part of the tube to remove the excess fluid and subcultured onto Muller-Hinton agar [10].

Paper discs with predetermined amounts of antibiotics were used. The following antimicrobial agents were tested: gentamycin $(10 \mu \mathrm{g})$, oxytetracyline $(30 \mu \mathrm{g})$, colistin $(10 \mu \mathrm{g})$, ciprofloxacin $(5 \mu \mathrm{g})$, doxycycline $(30 \mu \mathrm{g})$, nalidixic acid $(30 \mu \mathrm{g})$, co-trimoxazole (trimethoprim-sulfamethoxazole) $(1.25 / 23.75 \mu \mathrm{g})$, norefloxacin $(10 \mu \mathrm{g})$, lincospectin $(15 / 200 \mu \mathrm{g})$ and cefuroxime $(30 \mu \mathrm{g})$. All antibacterial discs were provided from Padtan Teb Company (Tehran, Iran). Following the application of antimicrobial discs, the plates were incubated at $37^{\circ} \mathrm{C}$ for 24 hours.

The diameters of the zones of inhibition were measured (millimetres) and were compared to internationally 
accepted measurements to determine the susceptibility or resistance of the isolates. Results were interpreted to the Clinical Laboratory Standard Institute (CLSI) guidelines [10]. Drug resistance patterns of the organisms were determined at three levels: Susceptible (S), Intermediate (I) and Resistant (R). The numbers of isolates of Escherichi coli which showed S, I and R patterns were determined. The percentages of antimicrobial resistance of each pattern (S, I and R) of isolates were calculated and reported as the results.

\section{Results}

All the isolates of Escherichia coli showed resistance to many antibiotics and a pattern of multiple drug resistance was observed. The rates of resistance against nalidixic acid (100\%), colistin (99\%), oxytetracycline (96\%), doxycycline (95\%), ciprofloxacin (91\%), trimethoprim-sulfamethoxazole (89\%), norefloxacin (88\%), lincospectin (53\%), cefuroxime (50\%) and gentamycin (17\%) were observed.

The rastes of susceptibility against gentamyci (81\%), lincospectin (41\%), cefuroxime (16\%), trimethoprimsulfamethoxazole (10\%), norefloxacin (9\%), ciprofloxacin (7\%), oxytetracycline and doxycycline (3\%), colistin and nalidixic acid (0\%) were observed. Antibacterial resistances pattern of isolated Escherichia coli are shown in Table 1.

\section{Discussion}

Antibiotic susceptibility pattern of Escherichia coli isolated from chickens showed highest resistance to nalidixic acid $(100 \%)$ and followed resistace to colistin (99\%), oxytetracycline (96\%), doxycycline (95\%), ciprofloxacin (91\%), co-trimoxazole (89\%), norefloxacin (88\%), lincospectin (53\%), cefuroxime (50\%) and gentamycin (17\%) were observed. In the area of this study respiratory viral diseases of broilers like Newcastle disease and infectious bronchitis occurs and subsequently secondary bacterial diseases like Escherichia coli infection are frequently observed. For treatment of secondary infection generally antibiotics are used with or without antibiogram testing. Besides, two compounds of antibiotics for treatment of secondary infection are usually administrated. The following compounds of antibiotic are almost used: polypeptides, lincosamides, macrolides, aminoglycosides, tetracyclines, sulfonamides, fluoroquinolones and pleuromutilines. This abuse consumption of antibiotics produced a wide range of antibiotic resistance. Moniri and Dastehgoli (2007) investigated the antimicrobial resistance pattern of Escherichia coli isolated from chickens in Iran. They observed a high level of resistance among the Escherichia coli as following: trimethoprim-sulfamethoxazole (98.7\%), ciprofloxacin (69.7\%), doxycycline (99.4\%) and nalidixic acid (99\%) [9]. The results of Moniri and Dastehgoli (2007) are similar to our finding in this study. Antibiotics are used as feed additives to improve feed efficiency and weight gain [11]. Many antibiotics are also used in feed and water to control disease. Indiscriminate use of antibiotics has provided selective pressure for the emergence of drug resistant strains of bacteria associated with poultry products [11]-[13]. Transmission of the R-plasmid from E. coli of poultry to human occurs very commonly [14]. Earlier studies revealed that use of fluoroquinolones in poultry was not appropriate due to the cross-resistance with fluoroquinolones used to treat human enteric infections. High resistance to chlortetracycline and oxytetracycline is of major concern because of the use of the same antibiotics in human medicine and poultry or in other food animals and the emergence of drug-resistant human pathogen [15] [16]. Antibiotic resistance of fecal E. coli was greater in broilers and turkeys that received antibiotics relatively frequently compared to layers, which had little exposure to antibiotics [14]. Similar antibiotic resistance patterns were present in E. coli isolated from people who worked with these birds, and in some instances specific strains were shared among the birds and workers in-

Table 1. Antibacterial resistance patterns among Escherichia coli isolated from chickens with colibacillosis (100 isolates).

\begin{tabular}{ccccccccccc}
\hline & \multicolumn{10}{c}{ Antibacterial agent } \\
\hline Results & GM10 & T30 & CL10 & CP5 & D30 & NA30 & SXT & NOR10 & LP & XM30 \\
\hline S (\%) & 81 & 3 & 0 & 7 & 3 & 0 & 10 & 9 & 41 & 16 \\
I (\%) & 2 & 1 & 1 & 2 & 2 & 0 & 1 & 3 & 6 & 34 \\
R (\%) & 17 & 96 & 99 & 91 & 95 & 100 & 89 & 88 & 53 & 50 \\
Total (\%) & 100 & 100 & 100 & 100 & 100 & 100 & 100 & 100 & 100 & 100 \\
\hline
\end{tabular}

GM10, gentamycin; T30, oxytetracyline; CL10, colistin; CP5, ciprofloxacin; D30, doxycycline; NA30, nalidixic acid; SXT, co-trimoxazole (trimethoprim-sulfamethoxazole); NOR10, norefloxacin; LP, lincospectin; XM30, cefuroxime; S, susceptible; I, intermediate; R, resistant. 
dicating that transmission of resistant organism and/or plasmids from poultry to people occurs commonly [14] [17].

\section{Conclusion and Recommendations}

Broiler chickens are main sources of protein for humans and broilers are very susceptible to Eschericia coli infection. Good management should be prepared for chickens farms to avoid secondary bacterial infection. Escherichia coli isolated from cases of colibacillosis showed resistant to many antibiotics. Indiscriminate use of antibiotics in food animals including chickens should be avoided to decrease drug resistant Eschericia coli strains.

\section{References}

[1] Rajesh, C., Rao, V.D.P., Gomez-Villamandos, J.C., Shukla, S.K. and Banerjee, P.S. (2001) Diseases of Poultry and Their Control. International Book Distributing Company, India.

[2] Saif, Y.M., Fadly, A.M., Glisson, J.R., McDougald, L.R., Nolan, L.K. and Swayne, D.E. (2008) Diseases of Poultry. 12th Edition, Iowa State Press, Iowa.

[3] Thyagarajan, D. (2011) Diseases of Poultry. Satish Serial Publishing House, Delhi.

[4] Swayne, D.E., Glisson, J.R., Jackwood, M.W., Pearson, J.E. and Reed, W.M. (1998) A Laboratory Manual for the Isolation and Identification of Avian Pathogens. 4th Edition, American Association of Avian Pathologists, University of Pennsylvania, Kennett Square.

[5] Quinn, P.J., Markey, B.K., Carter, M.E., Donnelly, W.J. and Leonard, F.C. (2002) Veterinary Microbiology and Microbial Disease. Blackwell Science, Oxford.

[6] Bada-Alambedji, R., Fofana, A., Seydi, M. and Akakpo, A.J. (2006) Antimicrobial Resistance of Salmonella Isolated from Poultry Carcasses in Dakar (Senegal). Brizilian Journal of Microbiology, 37, 510-515. http://dx.doi.org/10.1590/S1517-83822006000400020

[7] Adesiyun, A., Offiah, N., Seepersadsingh, N., Rodrigo, S., Lashley, V. and Musai, L. (2007) Antimicrobial Resistance of Salmonella spp. and Escherichia coli Isolated from Table Eggs. Food Control, 18, 306-311. http://dx.doi.org/10.1016/j.foodcont.2005.10.013

[8] Roy, P., Purushothaman, V., Koteeswaran, A. and Dhillon, A.S. (2006) Isolation, Characterization, and Antimicrobial Drug Resistance Pattern of Escherichia coli Isolated from Japanese Quail and Their Environment. Journal of Applied Poultry Research, 15, 442-446. http://dx.doi.org/10.1093/japr/15.3.442

[9] Moniri, R. and Dastehgoli, K. (2007) Antimicrobial Resistance among Escherichia coli Strains Isolated from Healthy and Septicemic Chickens. Pakistan Journal of Biological Sciences, 10, 2984-2987. http://dx.doi.org/10.3923/pjbs.2007.2984.2987

[10] Quinn, P.J., Carter, M.E., Markey, B. and Carter, G.R. (1994) Clinical Veterinary Microbiology. Wolf Publishing, London.

[11] Bower, C.K. and Daeschel, M.A. (1999) Resistance Responses of Microorganisms in Food Environments. International Journal of Food Microbiology, 50, 33-44. http://dx.doi.org/10.1016/S0168-1605(99)00075-6

[12] Quednau, M., Ahrne, S., Petersson, A.C. and Molin, G. (1998) Antibiotic Resistant Strains of Enterococcus Isolated from Swedish and Danish Retailed Chicken and Pork. Journal of Applied Microbiology, 84, 1163-1170. http://dx.doi.org/10.1046/j.1365-2672.1998.00463.x

[13] Scioli, C., Espostito, S., Anzilotti, G., Pavone, A. and Pennucci, C. (1983) Transferable Drug Resistance in Escherichia coli Isolated from Antibiotic-Fed Chickens. Poultry Science, 62, 382-384. http://dx.doi.org/10.3382/ps.0620382

[14] Van den Bogaard, A.E., London, N., Driessen, C. and Stobberingh, E.E. (2001) Antibiotic Resistance of Faecal Escherichia coli in Poultry, Poultry Farmers and Poultry Slaughterers. Journal of Antimicrobial Chemotherapy, 47, 763-771. http://dx.doi.org/10.1093/jac/47.6.763

[15] Bates, J., Jordens, J.Z. and Griffiths, D.T. (1994) Farm Animals as a Putative Reservoir for Vancomycin Resistant Enterococcal Infections in Man. Journal of Antimicrobial Chemotherapy, 34, 507-514. http://dx.doi.org/10.1093/jac/34.4.507

[16] Bager, F., Madsen, M., Christensen, J. and Aarestrup, F.M. (1997) Avoparcin as a Growth Promoter Is Associated with the Occurrence of Vancomycin-Resistant Enterococcus faecium on Danish Poultry and Pig Farms. Preventive Veterinary Medicine, 31, 95-112. http://dx.doi.org/10.1016/S0167-5877(96)01119-1

[17] Al-Ghamdi, M.S., El-Morsy, F., Al-Mustafa, Z.H., Al-Ramadhan, M. and Hanif, M. (1999) Antibiotic Resistance of Escherichia coli Isolated from Poultry Workers, Patients and Chicken in the Eastern Province of Saudi Arabia. Tropical Medicine and International Health, 4, 278-283. http://dx.doi.org/10.1046/j.1365-3156.1999.00392.x 